\title{
Pictorial rehearsal
}

\author{
MICHAEL J. WATKINS, ZEHRA F. PEYNIRCIOĠLU, and DOUGLAS J. BREMS \\ Rice University, Houston, Texas
}

\begin{abstract}
Subjects were presented with a sequence of picture-word items. The pictures were line drawings of common objects, and the words were the names of the objects. Half of the items were followed quickly by the next item, and the other half by a 15-sec "rehearsal interval." The subjects were told to use these intervals to rehearse the item just presented, either by saying the word over and over to themselves or by maintaining the picture before the mind's eye. There followed a test in which each item from the study list was cued with a fragment of either the picture or the name. Consistent with previous findings, identification was more probable for fragments of items that had been followed by a rehearsal interval. In addition, this advantage was found to be greater when the type of cue matched the mode of rehearsal that the subject had been instructed to adopt; there was in fact little, if any, advantage of rehearsal when cue and mode of rehearsal mismatched. This pattern of results suggests functionally distinct pictorial and verbal modes of rehearsal that serve not only to maintain information in conscious mind but also to build up memory proper.
\end{abstract}

Covert or mental rehearsal can be an effective means of acquiring information, and largely for this reason it has become a central construct in memory theories and the subject of a great deal of laboratory research (Johnson, 1980). In the overwhelming majority of cases, the rehearsed materials in this research have been verbal, and a widely held assumption that rehearsal takes a verbal, and more specifically, a speech-like, form has provided a satisfactory interpretation of the data. What is not clear is whether rehearsal can take other forms-whether, for example, the acquisition of information can be promoted by a form of covert rehearsal that can reasonably be characterized as pictorial.

As it happens, a few studies have investigated the role of rehearsal in memory for nonverbal materials, mostly pictorial items such as line drawings and photographs. Dominating this research is a paradigm in which, in its simplest form, a sequence of pictures is presented with blank intervals interpolated after some, but not all, of the pictures, and then, after all pictures have been presented, a recognition test is given. With occasional exceptions (Bird \& Cook, 1979; Shaffer \& Shiffrin, 1972), this research has revealed a reliably higher level of recognition for those pictures followed by a blank interval than for those followed directly by the next picture in the sequence (Hines \& Smith, 1977; Intraub, 1979; Lutz \& Scheirer, 1974; Read, 1979; Tversky \& Sherman, 1975; Weaver, 1974; Weaver \& Stanny, 1978). Moreover, this beneficial effect has been shown to be under the subject's control, and therefore may be said to arise from a deliber-

This research was supported by National Institute of Mental Health Grant MH35873 to the first author. The authors thank Welli Yeh for her help with the manuscript. The second author is now at Boğaziçi University, Istanbul, Turkey. Requests for reprints should be sent to Michael J. Watkins, Department of Psychology, Rice University, Houston, TX 77251. ate strategy of rehearsal rather than from passive consolidation of the last-presented picture during the blank interval. Thus, when two or more pictures are presented, either simultaneously or in quick succession, and then one (or some subset) of them is cued for rehearsal, the cued picture(s) will be more likely to be identified in a later recognition test than will the uncued picture(s) (Graefe \& Watkins, 1980; Watkins \& Graefe, 1981).

Although these studies demonstrate that rehearsal can be an effective means of remembering pictorial material, they do not demonstrate that the rehearsal is of a pictorial nature. Perhaps the observed effect is due to rehearsal that is no different from the speech-like rehearsal presumed to occur for verbal material. The improvement in picture identification could have arisen from rehearsal of verbal labels or descriptions of the pictures rather than from rehearsal of pictorial images.

To be sure, some of the picture-rehearsal studies have obtained a reliable rehearsal effect with pictures that were not easy to label (e.g., Hines \& Smith, 1977) or with the usefulness of simple labels undercut by the inclusion in the recognition test of distractor pictures that shared the same labels as the target pictures (Read, 1979; Tversky \& Sherman, 1975; Weaver, 1974; Weaver \& Stanny, 1978). But such precautions hardly rule out a verbal interpretation of the observed rehearsal effects; after all, a statistically significant effect can occur if just some subjects apply just partially adequate verbal descriptions to just some items. In short, there is currently no clear evidence that learning can occur through a nonverbal mode of rehearsal.

The purpose of this article is to report an experiment designed to address this issue, and specifically the question of whether memory, as measured in a subsequent test, can be enhanced by a pictorial as well as by a verbal mode of rehearsal. Subjects were presented with a series of 
picture-name items, some of which were followed directly by the next item and others by a "rehearsal interval." Half of the subjects were encouraged to use these intervals to rehearse the name of the just-presented item, and the other half to rehearse the picture. If rehearsal were functionally equivalent for the two groups, then its effect, as indicated in subsequent memory tests, would be expected to be the same. If, on the other hand, the two groups really were using functionally different modes of rehearsal, then the effect on performance might well differ in a way that depended on the type of test. Presumably, one group would rehearse in a way that would selectively enhance memory for the verbal aspect of the items, whereas the other group would rehearse in a manner that would selectively enhance memory for the pictorial aspect. To distinguish between these two possibilities, all the subjects were given two kinds of memory tests, one that could reasonably be assumed to be specifically sensitive to the verbal aspect of the studied items, and the other to the pictorial aspect. More concretely, one test called for identification of fragments of the names, and the other for identification of fragments of the pictures. At issue was whether the effect of rehearsal interval depended on whether the way in which the subjects had been instructed to rehearse matched or mismatched the type of fragment used in the identification test.

\section{METHOD}

\section{Materials}

Fifty-two stimulus pictures were used, 4 for practice and 48 in the experiment proper. The pictures were simple line drawings of familiar objects; examples are shown in the upper panel of Figure 1. The pictures were presented by means of a slide projector, with an area of projection of approximately $2 \mathrm{ft}$ square, and the subjects were seated 8 to $16 \mathrm{ft}$ from the screen. The names of the objects were presented from a tape recorder.

The fragments used in the test phase of the experiment were of two kinds, word fragments and picture fragments. A word fragment included approximately half of the letters in the word, with dashes in place of the missing letters. Choice of letters for inclusion in the fragments was such that, from among the 52 words used in the experiment, each fragment fitted only the word for which it was intended. The picture fragments were obtained by overlaying the pictures with Letratone LT 163 transparency, which resembles a wall of opaque bricks fixed by transparent mortar; in addition, these fragments were presented out of focus, the extent of defocusing having been determined on an individual picture basis in pilot research. Examples of the two types of fragments are shown in the middle and lower panels of Figure 1.

\section{Design}

The subjects were assigned randomly to two groups of equal size, which may be called the verbal rehearsal group and the pictorial rehearsal group.

Both groups were presented with the same sequence of 32 picturename items, which were chosen randomly from the main 48 -item set. For any given subject, a randomly selected half of these 32

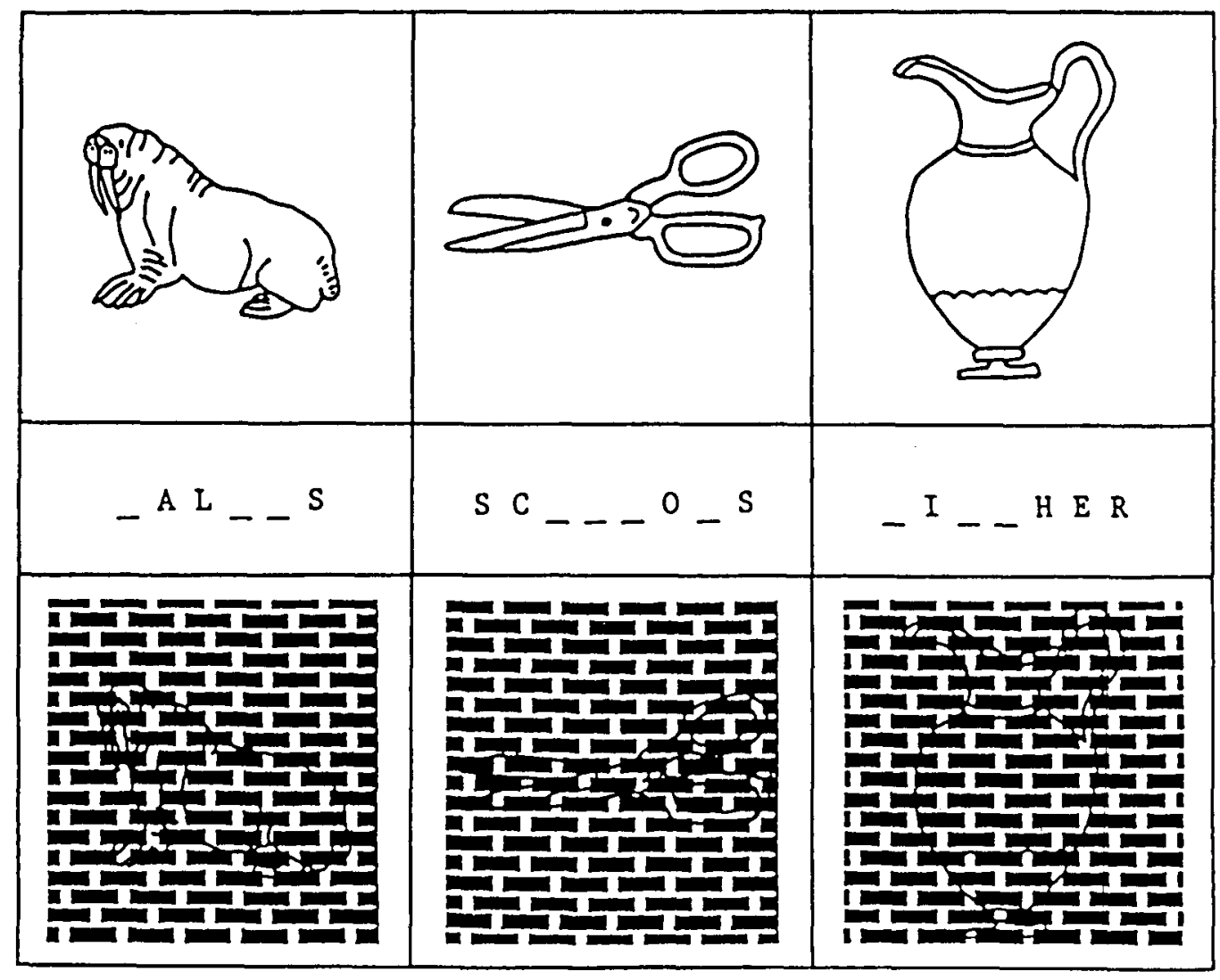

Figure 1. Examples of the pictures used in the study phase of the experiment (upper panel), and of the word fragments (middle panel) and picture fragments (lower panel) used in the test phase. The picture fragments were presented out of focus. 
items were presented in the rehearsal condition, and the other half in the nonrehearsal condition. The assignment of items to the rehearsal and nonrehearsal conditions was counterbalanced between subjects so that each picture occurred in each condition for an equal number of subjects within both the verbal and the pictorial rehearsal groups.

Consider now the fragment identification test. For any given subject, 8 of the 16 items that had been presented in the rehearsal condition and 8 of the 16 that had been presented in the nonrehearsal condition were cued with word fragments, and the remainder with picture fragments; similarly, 8 of the 16 new items (i.e., those that had not been presented at all in the study sequence) were cued with word fragments, and the other 8 were cued with picture fragments. The choice of which subset of 8 items were cued with which type of fragment was counterbalanced such that each item from the study sequence occurred in each combination of rehearsal condition and fragment type for a quarter of the subjects within each rehearsal group, and each new item was cued with each type of fragment for half of the subjects within each group.

In short, if we restrict consideration to the items in the study sequence, the experiment is of mixed design, with requested mode of rehearsal (verbal or pictorial) as a between-subjects variable and rehearsal condition (rehearsal or nonrehearsal) and type of fragment (word fragments or picture fragments) as within-subjects variables. The variables were combined factorially, with items being completely counterbalanced between combinations of conditions.

\section{Subjects}

The subjects were 64 Rice University students. They participated for pay or in fulfillment of a course requirement.

\section{Procedure}

The subjects were tested up to six at a time. They were told in some detail how a long sequence of picture-name items would be presented, with unfilled intervals occurring after some, but not all, of the items. These intervals were to be used to rehearse the justpresented item, and the form of this rehearsal was carefully described. The subjects in the verbal rehearsal group were told to concentrate on the name of the object and to mentally repeat it over and over; those in the pictorial rehearsal group were told to maintain an image of the picture throughout the interval and to scan the image continuously with the mind's eye.

Presentation of the main sequence was preceded by a practice sequence that included just four items. Each picture was displayed for $1.25 \mathrm{sec}$, and its name was given auditorily as the picture was shown on the screen. The first and fourth items of the practice sequence were followed by a 15 -sec blank interval and then, after a .75-sec change time, by the next item or by an end-of-sequence slide. The other two items were followed by the next item after just the change time. Ten seconds into each rehearsal interval, the subjects were signaled to rate on prepared response sheets their success in following the rehearsal instructions, using a 5-point scale ranging from "not at all well" (1) to "very well" (5); the purpose of this requirement was merely to encourage the subjects to take the rehearsal instructions seriously. Both the names of the items and the rehearsal-rating signal (the word "rate") were presented by means of a tape recorder. Note that the subjects did not know the rehearsal condition of an item until $.75 \mathrm{sec}$ after its presentation, so that any advantage that the rehearsal condition might show in fragment identification should not be attributed to a difference in the kind or amount of attention paid to the items during their actual presentation.

The practice sequence was followed by a practice test in which the subjects were presented with fragments of the two rehearsal items and of two new items in random order; note that there was no test of the two nonrehearsal items. The fragments were displayed for $10 \mathrm{sec}$ each and were all of a form corresponding to the mode of rehearsal the subjects had been instructed to adopt-word fragments for the verbal rehearsal group and picture fragments for the pic- torial rehearsal group. There was a blank interval of $5 \mathrm{sec}$ between fragments, which allowed the subjects to write the name of the item just cued. The subjects were led to expect that the practice studytest procedure would be a precise miniature version of that for the experiment proper, the intention here being to encourage the subjects both to restrict rehearsal to those items assigned to the rehearsal condition and to rehearse in the manner in which they had been instructed.

The presentation phase of the experiment proper was indeed merely a lengthier version of the practice presentation phase. The test, however, was different. This time, all of the presented items, the nonrehearsal as well as the rehearsal items, were cued. In addition, the test included both kinds of fragment cues, and not just the kind that the subjects had been led to expect. Just prior to the main test, the nature of the unfamiliar cue was explained and its use illustrated with items from the practice set. The experimenter explained that both picture-fragment cues and word-fragment cues would be presented, and that for each cue the answer could be a rehearsal, a nonrehearsal, or a new item. In all cases, their task was the same: to try to identify the items of which the cues were fragments. Timing was as in the practice phase.

\section{RESULTS}

Figure 2 summarizes the results in terms of the percentage of fragments identified. The upper panel shows how, for each rehearsal group, picture fragment identification varied according to whether the items being cued had been rehearsed, had been presented but not rehearsed, or were new; the lower panel does the same thing for word fragment identification. Of primary concern are the data for the nonrehearsal and rehearsal conditions. As Figure 2 shows, these data conform rather well to the hypothesis of separate pictorial and verbal modes of rehearsal, for they indicate that allowing a subject to rehearse a picturename item had a greater effect on later identification of a fragment of the picture or of the name when the type of rehearsal the subjects were instructed to adopt was consistent with the type of fragment used. Thus, as the upper panel of Figure 2 shows, when the fragments in the identification test were of pictures, the advantage of the rehearsal condition over the nonrehearsal condition was greater for the pictorial rehearsal group than for the verbal rehearsal group. And conversely, as the lower panel shows, when the fragments were of words, the advantage of the rehearsal condition was greater for (in fact, was confined to) the verbal rehearsal group. Notice that, for both types of fragments, mode of rehearsal mattered for the rehearsal items but not for the nonrehearsal items.

These observations were confirmed by statistical analyses. Most importantly, an analysis of variance (with requested mode of rehearsal as a between-subjects variable, and rehearsal condition and type of fragment as within-subjects variables) on data for the rehearsal and nonrehearsal items revealed a reliable second-order interaction $[F(1,62)=16.43, p<.001]$. More specific analyses show that this interaction can be described in terms of rehearsal's being more advantageous when the test fragments were of the kind expected: An analysis of the data for just the rehearsal items yielded a reliable interaction between mode of rehearsal and type of fragment 


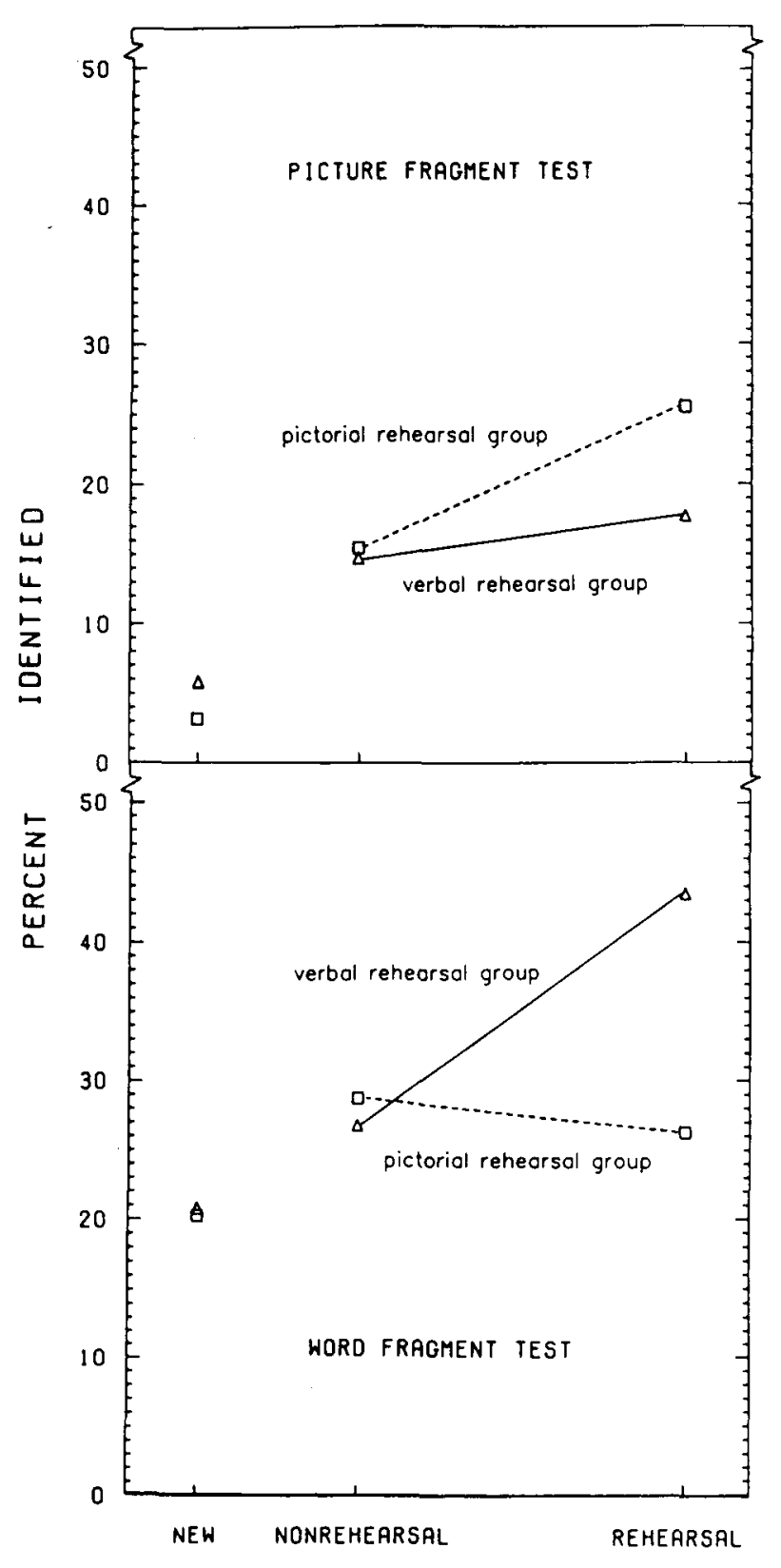

CONDITION OF TEST ITEM

Figure 2. Mean percentage of picture and word fragments of rehearsal, nonrehearsal, and new items correctly completed by the pictorial and verbal rehearsal groups.

$[F(1,62)=12.50, p<.001]$, whereas in a similar analysis of the data for nonrehearsal items, this interaction was not reliable $[F(1,62)=0.06, p>.10]$. More particularly, performance in the rehearsal condition exceeded that in the nonrehearsal condition both when subjects expected and received picture fragments $[\mathrm{t}(31)=2.63$, $\mathrm{p}<.02$ ] and when they expected and received word fragments $[t(31)=5.09, \mathrm{p}<.001]$; by contrast, the rehearsal condition held no reliable advantage either when subjects expected word fragments and received picture fragments $[t(31)=1.49, p>.10]$ or when they expected picture fragments and received word fragments [t $\mathrm{t}(31)=$ $-0.76, p>.10]$.

Apart from the second-order interaction, the overall analysis of the data for the presented items showed reliable main effects for rehearsal $[\mathrm{F}(1,62)=20.24, \mathrm{p}<$ $.001]$ and type of fragment $[F(1,62)=27.73, p<.001]$, reflecting a higher level of identification of rehearsal than of nonrehearsal items and for word fragments than for picture fragments. There was no reliable effect of mode of rehearsal $[F(1,62)=0.42, p>.10]$. Type of fragment interacted with mode of rehearsal $[\mathrm{F}(1,62)=5.50$, $\mathrm{p}<.05]$, which means that although mode of rehearsal reliably affected only the rehearsal items, the diluted effect that remained when the data for the nonrehearsal items were added in was statistically significant. The interaction of mode of rehearsal with rehearsal condition was of borderline reliability $[\mathrm{F}(1,62)=3.86, \mathrm{p}=.06]$ and can be described in terms of a bigger effect of rehearsal condition for the verbal rehearsal group than for the pictorial rehearsal group. Type of fragment did not interact with rehearsal condition $[\mathrm{F}(1,62)=0.03, \mathrm{p}>.10]$.

Although less relevant to the question of primary concern, performance on items not presented in the study list should be noted. As Figure 2 shows, these items were less likely to be identified than were presented items. Analyses confirmed that identification was less probable for new items than for the nonrehearsal items [ts(63) = 5.09 and 2.97 for the picture and word fragment tests, respectively] or for the rehearsal items $[\operatorname{ts}(63)=7.33$ and 5.20 , respectively] ( $\mathrm{p}<.01$ in all cases). Analysis of the new-item data revealed a reliable main effect for type of fragment $[F(1,62)=37.45, p<.001]$, reflecting a higher level of identification in the word fragment test. Neither the effect of mode of rehearsal $[F(1,62)=0.23, p>.10]$ nor the interaction between mode of rehearsal and type of fragment $[F(1,62)=0.36, p>.10]$ was reliable. This last result, along with the lack of a corresponding interaction in the data for the nonrehearsal items, supports the conclusion that the critical second-order interaction for the rehearsal and nonrehearsal data had its origin in the rehearsal intervals, and, more specifically, that during these intervals the subsequent effectiveness of the two types of fragments increased to an extent that depended on mode of rehearsal.

\section{CONCLUSION}

Our findings support the notion that rehearsal can take a pictorial as well as a verbal form. In particular, instructing the subjects to rehearse in a pictorial fashion enhanced their likelihood of identifying a fragment of a rehearsed picture but had no beneficial effect whatsoever on the identification of a fragment of the name of the object depicted by the picture. Consistent with these findings are those from a variety of other studies usually taken, through reasoning similar to our own, as indicating that information can be held in the conscious mind in a form that can 
be either speech-like or pictorial (e.g., Brooks, 1968; Peterson, Thomas, \& Johnson, 1977). But the present findings go beyond the demonstration of two different forms of mental representation, for they tell us that each form can serve in its own functionally distinct way not only as a means of temporarily retaining information in mind, but also as a basis of a rehearsal that can affect behavior long after such retention.

\section{REFERENCES}

BIRD, J. E., \& Cook, M. (1979). Effects of stimulus duration and ISI on accuracy and transference errors in pictorial recognition. Memory \& Cognition, 7, 469-475.

Brooks, L. R. (1968). Spatial and verbal components of the act of recall. Canadian Journal of Psychology, 22, 349-368.

Graefe, T. M., Watkins, M. J. (1980). Picture rehearsal: An effect of selectively attending to pictures no longer in view. Journal of Experimental Psychology: Human Learning and Memory, 6, 156-162.

HiNEs, D., \& SMITH, S. (1977). Recognition of random shapes followed at varying delays by attended or unattended shapes, digits, and line grids. Journal of Experimental Psychology: Human Learning and Memory, 3, 29-36.

INTRAUB, H. (1979). The role of implicit naming in pictorial encoding.
Journal of Experimental Psychology: Human Learning and Memory, $5,78-87$.

Johnson, R. E. (1980). Memory-based rehearsal. In G. Bower (Ed.), The psychology of learning and motivation (Vol, 14, pp. 263-307). New York: Academic Press.

LUTZ, W. J., \& SCHEIRER, C. J. (1974). Coding processes for pictures and words. Journal of Verbal Learning and Verbal Behavior, 13, 316-320.

Peterson, M. J., Thomas, J. E., \& Johnson, H. (1977). Imagery, rehearsal, and the compatibility of input-output devices. Memory \& Cognition, 5, 415-422.

READ, J. D. (1979). Rehearsal and recognition of faces. American Journal of Psychology, 92, 71-85.

Shaffer, W. O., \& ShIFFrin, R. M. (1972). Rehearsal and storage of visual information. Journal of Experimental Psychology, 92, 292-296.

TVERSKY, B., \& SHERman, B. (1975). Picture memory improves with longer on time and off time. Journal of Experimental Psychology: Human Learning and Memory, 1, 114-118.

WatKINS, M. J., GRAEFE, T. M. (1981). Delayed rehearsal of pictures. Journal of Verbal Learning and Verbal Behavior, 19, 46-53.

WeAVER, G. E. (1974). Effects of poststimulus study time on recognition of pictures. Journal of Experimental Psychology, 103, 799-801.

Weaver, G. E., \& StanNy, C. J. (1978). Short-term retention of pictorial stimuli as assessed by a probe recognition technique. Journal of Experimental Psychology: Human Learning and Memory, 4, 55-65.

(Manuscript received June 25, 1984;

revision accepted for publication October 17, 1984.) 